\title{
Hijacking cellular signals
}

\author{
Peter Nick $^{1}$
}

Received: 4 October 2017 / Accepted: 4 October 2017 / Published online: 11 October 2017

(C) Springer-Verlag GmbH Austria 2017

Around half of organisms on this planet are thought to follow a parasitic lifestyle (Zimmer 2001). Parasites are not only relevant as pathogens, but also as selective factors that link different organisms into a common evolutionary relationship, which can cross even the borders of biological kingdoms. The complexity and sophistication of parasitic manipulation is breathtaking and at first sight hard to explain. At a second glance, parasitic interactions are found to hitchhike on the complex signaling regulating the response of the host to the challenges of environment or to the modulations imposed by development. Parasites often simulate these signals to elicit some responses (those that are needed to proceed in their own life cycle), while not activating the full set of responses that would be activated by the true host signal. An alternative strategy seems to release a signal that triggers a host response at a condition, where this response is not appropriate. Both strategies can be understood as hijacking a signal by shifting it out of context-in other words, parasitism works by undermining the communication system of the host. So far, humans control parasites mainly by poisoning them with devastating ecological footprints and often collateral damage to the host itself or to other organisms. If one follows the evolutionary logics of parasitism, it might be a more promising and sustainable strategy to corrupt its own signaling. However, to develop such strategies, we have to know the details. These details are often difficult to observe, because the events are not displayed openly to the observer, but proceed inside an organism. Three contributions to the current issue highlight, for

Peter Nick

peter.nick@kit.edu

1 Molecular Cell Biology, Botanical Institute, Karlsruhe Institute of Technology, Fritz-Haber-Weg 4, 76131 Karlsruhe, Germany different plant parasites, advances in their observation, the analysis of their strategies, and in their control by "hijacking the hijacker."

The contribution by Ohtsu et al. (2017) in the current issue reports a methodological breakthrough, which allows to see what so far has remained hidden inside a plant root infected by parasitic nematodes. These animals can invade a root and induce there a feeding structure with peculiar cellular features, such as giant cells with numerous nuclei, and specific structures on the cell wall. To observe those in the root interior in a non-invasive manner is very difficult indeed, such that most knowledge about these syncytia comes from histological studies (Golinowski et al. 1996). Using a clearing solution in combination with two-photon imaging, the authors have now succeeded to follow the development of feeding structures in soybean. They could observe that the structure of the syncytium in the cortex differed with respect to secondary wall structures from the syncytium formed in the vascular tissue. This means that specific features of the host tissue are playing a role for the morphogenesis of the syncytium and therefore provides evidence for an ongoing developmental process that is modulated by the parasite. This is consistent with a working hypothesis, where the cellular features of the syncytium have been associated with transfer cells that function in transport during regular development (Gunning and Pate 1969).

The evolutionary arm race between host and pathogen leads to an interaction that becomes progressively specific culminating in a situation, where a specific strain of the parasite can only infect a specific genotype of the host. On the other hand, a parasite often requires several hosts to complete its life cycle, adding a further level of complexity. A case study on the rust fungus Puccinia striiformis f. sp. tritici by Guo et al. (2017) in the current issue highlights this issue as one of the economically most relevant fungal parasites. The breeding of resistant wheat varieties has been a very 
successful approach to control this pathogen without the need for extensive fungicide treatment. Since this fungus was thought to be strictly asexual, the risk of evolutionary changes that might break this resistance was thought to be negligible. This view was shattered by the detection of sexual stages on different host plants such as barberry (Berberis), because this would mean that the parasite can undergo rapid change by making use of a sexual phase taking place on a different host. Strangely enough, barberry shows rarely any symptoms after infection with spores of the pathogen. The authors can now show that this is caused by efficient non-host resistance including rapid oxidative burst, formation of callose plugs underneath sites, where the fungus attempts to penetrate the cell, and by efficient suicide (so called hypersensitive response) of the infected cells. As a result, the parasite, although attempting to pursue its life cycle, can produce only abnormal structures, such as deformed substomatal vesicles or germ tubes and only inefficiently draws energy from this "non-host," due to a reduced number of haustoria. It remains to be investigated, whether the stress imposed by this efficient non-host resistance of barberry is stimulating the transition towards a sexual cycle creating the risk to create new parasite strains that might damage the success story of resistance breeding in wheat. In that case, the resistance of one (non)-host would be "paid" by eroded resistance in the other host plant.

The close link between parasitism and hijacked signals is illustrated, possibly in its purest manifestation, in viral interactions. A virus is not even alive, but rather resembles a life form reduced to its informational aspect. In their comprehensive review on Papaya leaf curl, Varun et al. (2017) in the current issue not only consider the biogeographic history, the molecular evolution, and the mechanisms of virulence for this most important viral disease of Papaya with devastating impact on the economy of many tropical countries. They also consider approaches to control and contain this disease. Since it is not possible to kill something, which is not alive, curative measures are of limited success here. Is there anything beyond suppressing the viral vectors (white fly) by insecticides or production of virus-free plant clones by tissue culture? An interesting approach would be to fight information with information. If the virus hijacks host transcription using bidirectional reading frames encoding six products, a strategy of containment could be to target those by a miRNA approach, such that the resulting double-stranded RNA can be recognized by the host and silenced. This strategy to "hijack the hijacker" has been successful in other viral diseases and might be effective also in case of Papaya, a crop that can be efficiently engineered genetically.

\section{Compliance with ethical standards}

Conflict of interest The author declares that there is no conflict of interest.

\section{References}

Golinowski W, Grundler FMW, Sobczak M (1996) Changes in the structure of Arabidopsis thaliana during female development of the plant-parasitic nematode Heterodera schachtii. Protoplasma 194: 103-116

Gunning BES, Pate JS (1969) "Transfer cells" plant cells with wall ingrowths, specialized in relation to short distance transport of solutes - their occurrence, structure, and development. Protoplasma 68:107-133

Guo J, Jiao M, Tan C, Guo J, Wang L, Zhang H, Kang Zh (2017) Basidiospores of Puccinia striiformis f. sp. tritici succeed to infect barberry, while urediniospores are blocked by non-host resistance. Protoplasma. https://doi.org/10.1007/s00709-017-1114-z

Ohtsu M, Sato Y, Kurihara D, Suzaki T, Kawaguchi M, Maruyama D, Higashiyama T (2017) Spatiotemporal deep imaging of syncytium induced by the soybean cyst nematode Heterodera glycines. Protoplasma. https://doi.org/10.1007/s00709-017-1105-0

Varun P, Ranade SA, Saxena S (2017) A molecular insight into papaya leaf curl-a severe viral disease. Protoplasma. https://doi.org/10. 1007/s00709-017-1126-8

Zimmer C (2001) Parasite rex: inside the bizarre world of nature's most dangerous creatures. Atria, New York 